\title{
Three-Dimensional Echocardiography in Congenital Heart Disease
}

\author{
John M. Simpson · Kuberan Pushparajah
}

Published online: 6 February 2013

(c) Springer Science + Business Media New York 2013

\begin{abstract}
Three-dimensional echocardiography has an increasing role in the management of patients with congenital heart disease. Technical advances mean the technique can be applied from fetal life through to adulthood. Clinical applications relate both to the assessment of morphology and cardiac function. This is being applied to diagnostic evaluation, serial follow up and procedural guidance in the catheterization laboratory and operating room. Developments in this field are reviewed with emphasis on recent data and future perspectives.
\end{abstract}

Keywords Three-dimensional echocardiography ·

Congenital heart disease $\cdot$ Morphology $\cdot$ Pediatrics .

Cardiac function $\cdot$ Fetal cardiology $\cdot$ Ventricular function

$\begin{array}{ll}\text { Abbreviations } \\ \text { SVC } & \text { Superior vena cava } \\ \text { ASD } & \text { Atrial septal defect } \\ \text { LA } & \text { Left atrium } \\ \text { RA } & \text { Right atrium }\end{array}$

John M. Simpson and Kuberan Pushparajah contributed equally to this study.

J. M. Simpson $(\varangle) \cdot$ K. Pushparajah

Department of Congenital Heart Disease, Evelina Children's

Hospital, 6th Floor, Guy's and St Thomas' NHS Trust,

London SE1 7EH, UK

e-mail: john.simpson@gstt.nhs.uk

J. M. Simpson · K. Pushparajah

Division of Imaging Sciences and Biomechanical Engineering,

Rayne Institute, King's College London, St Thomas' Hospital,

London SE1 7EH, UK

\section{Introduction}

Three-dimensional echocardiography (3DE) has emerged as an integral part of the assessment of patients with congenital heart disease [1-3]. Advances in probe design, with the introduction of real-time "matrix" probes, coupled with increased computer processing power and improved software for interrogation of data have all contributed to the acceptance of the technique into mainstream practice. The availability of a wide range of three-dimensional (3D) transducers, including pediatric and transesophageal probes mean that the technique can be applied in a variety of settings ranging from assessment of small infants and children through to live guidance of interventional catheterization procedures.

A major challenge for those undertaking 3DE, particularly in the setting of congenital heart disease, is how best to present the acquired images so that the novel projections which can be achieved are readily interpretable by those who would value the additional information, such as cardiac surgeons and interventional cardiologists. Within the past year, publications have emerged which discuss this issue either specifically for congenital heart disease [4•] or as part of wider 3DE recommendations which incorporate congenital heart disease [5•]. Areas of application of 3DE which remain prominent include insights into congenital lesions, such as atrioventricular valves or complex cardiac morphology, where the findings have a direct impact on surgical approach or technique. Interventional cardiac catheterization is increasingly being guided by transesophageal 3DE, and recent published work has focused on such applications such as interventional closure of ventricular septal defects (VSDs) and "difficult" atrial septal defects (ASDs).

The application of 3DE for the assessment of cardiac function in congenital heart disease continues to expand, 
and is being critically compared to other techniques such as cardiac magnetic resonance imaging (CMRI). Assessment of ventricular dyssynchrony is also being investigated in children, which is being assisted by the publication of normal reference ranges and application to both the left ventricle (LV) and right ventricle $(\mathrm{RV})$. This review aims to summarize the advances which have occurred recently and the areas of ongoing research.

\section{Standardization of 3D Echocardiography}

Within the past year, joint American and European guidelines have been published which provide a review and recommendations for the acquisition and display of $3 \mathrm{D}$ echocardiographic images [5•]. The document comprehensively reviews technical aspects of $3 \mathrm{DE}$, different acquisition modalities, and optimization of image acquisition. There are some aspects of image presentation which might not be universally adopted and some of these are particularly relevant to congenital practice. One of the central concepts which has been adopted in many congenital units relates to "anatomic" display of cardiac structures [6]. If this is extended into $3 \mathrm{DE}$ then our view has been that $3 \mathrm{DE}$ should be presented as far as possible in an anatomic projection, which was the basis for our recent publication in this area [4•]. Examples where our approach deviates from the recommendations of the recent standards document includes the imaging projection of the mitral, tricuspid and aortic valves. With respect to the tricuspid valve, for example, the standards document projects the ventricular septum horizontally when imaging the valve from the ventricular aspect, whereas our preferred approach rotates the tricuspid valve so that the diaphragmatic surface is lowermost, in keeping with the true anatomic orientation of the valve. In a similar fashion, we prefer orientation of the aortic valve with the right coronary cusp uppermost, akin to the surgical view, and orientation of the view of the mitral valve to a more anatomic position. Although these differences in orientation approach may appear relatively minor, they are important in congenital practice where the relative positions of cardiac structures cannot be taken for granted and where we believe that the imaging projection should be intuitive.

\section{Cardiac Morphology}

\section{Atrioventricular Valves}

Valvar lesions are generally regarded as some of the most suitable to be studied by 3DE. Our understanding of the atrioventricular septal defect (AVSD), both in terms of its morphology and, importantly, mechanisms of insufficiency following initial repair, has been the subject of recent detailed study. The morphology of the AVSD has been studied by transesophageal echocardiography (TEE) and compared to anatomic specimens, which have beautifully correlated the echocardiographic and pathological findings. The ability of 3DE to include the entire atrioventricular junction plus the anatomy and significance of the atrial and ventricular components of the defect was clearly demonstrated [7]. A recent review summarized the advantages and disadvantages of $3 \mathrm{DE}$ compared to cross-sectional echocardiography alone [8]. A crucial aspect that was emphasized was the utility of 3DE to image the chordal and papillary muscle support apparatus of the left atrioventricular valve. This has been extended recently into a quantitative study of the left atrioventricular valve by $3 \mathrm{DE}$ assessment of the annulus and the geometry of the subvalvar apparatus [9]. The 3DE approach to the subvalvar apparatus is not restricted to the AVSD and has been extended to other lesions such as the parachute mitral valve [10]. The cropping techniques, particularly that of cropping across the plane of the anterior leaflet of the left atrioventricular valve, has the potential to expose the subvalvar apparatus in a way which is not possible using crosssectional techniques.

\section{The Aortic Valve}

New 3DE data have not been restricted to atrioventricular valves, and the aortic valve and root has been the subject of several recent studies. The utility of $3 \mathrm{DE}$ to image the anatomic variability in bicuspid and other abnormal aortic valves has been reported $[11,12]$, as has the ability of the technique to measure the true maximum diameter of the aortic root in children with aortic root dilatation [13]. When balloon dilation of the aortic valve is considered, 3DE provides accurate information relating to annulus dimension and effective aortic valve orifice area [14, 15]. Although the data relating to utility of $3 \mathrm{DE}$ to image the aortic valve morphology and size are encouraging, visualization of the aortic valve in smaller infants with faster heart rates remains challenging. This particularly relates to imaging of the whole valve cusp rather than simply the commissures. Drop-out of signal in the belly of the valve cusps remains an issue.

\section{Complex Cardiac Abnormalities}

Complex abnormalities of the cardiac connections are a particular challenge to those involved in congenital practice. Double outlet RV has a number of challenges, including the relative position of the great arteries, location and size of the interventricular communication, and feasibility of "routing" from the LV to the aorta. Our group has 
recently described a systematic approach to this lesion to assist in surgical planning [16], which involves cropping to view the AV connections, VSD and great arteries from the ventricular aspect coupled with projections from the RV to show the position and size of the VSD.

\section{Catheter Intervention}

Visualization of the atrial septum by $3 \mathrm{DE}$ has assumed great importance because of the ability to assess the location, size, and shape of the ASD [17] prior to device closure in selected cases [18] (Fig. 1a). Previous work has demonstrated the morphological differences between defects [19], but a more recent study has provided a critical analysis of the role of 3DE in predicting device selection and comparison with twodimensional echocardiography (2DE) and balloon-sizing [20]. That study showed that 3DE was an effective means of measuring ASD's rather than using 2DE with a different angle of rotation in finding the maximal diameter, although there was a very slight underestimation of the maximal defect size by the $3 \mathrm{DE}$ technique compared to $2 \mathrm{DE}$. The $3 \mathrm{DE}$ is particularly suitable for ASD's of complex shape where live assessment of catheter and device position is paramount [21]. Use of a 3DE approach during assessment of ASD's prior to and during catheter closure requires the use of different 3DE modalities with which the operator needs to become familiar. Full volume acquisitions over multiple cardiac cycles provide a large field of view and help to identify rims and details of the morphology. However, truly live 3DE modes, including 3D zoom modes, are used to guide the procedure itself (Fig. 1b). Each of the modalities has specific strengths and weaknesses which has been reported recently in a large cohort of patients undergoing ASD occlusion [22].

Selected VSD's may also be closed by transcatheter means. Recent papers report on large cohorts of patients being treated by the method as well as the introduction of newer VSD occlusion devices which may avoid some of the complications that have been described, such as acquired complete heart block or damage to adjacent structures $[23,24]$. The $3 \mathrm{DE}$ can be used to define the position and morphology of VSD's [25] and is an effective means of guiding such interventions, with potential advantages related to device sizing, monitoring of catheter position, and device deployment [26]. The rims of perimembranous VSD's are crucially important, particularly those which are adjacent to the tricuspid and aortic valves. The size and shape of such rims can be readily appreciated by $3 \mathrm{DE}$ both before and after device deployment. At these authors' institution, 3DE is used routinely to assist with catheter occlusion of VSD's, which has included both
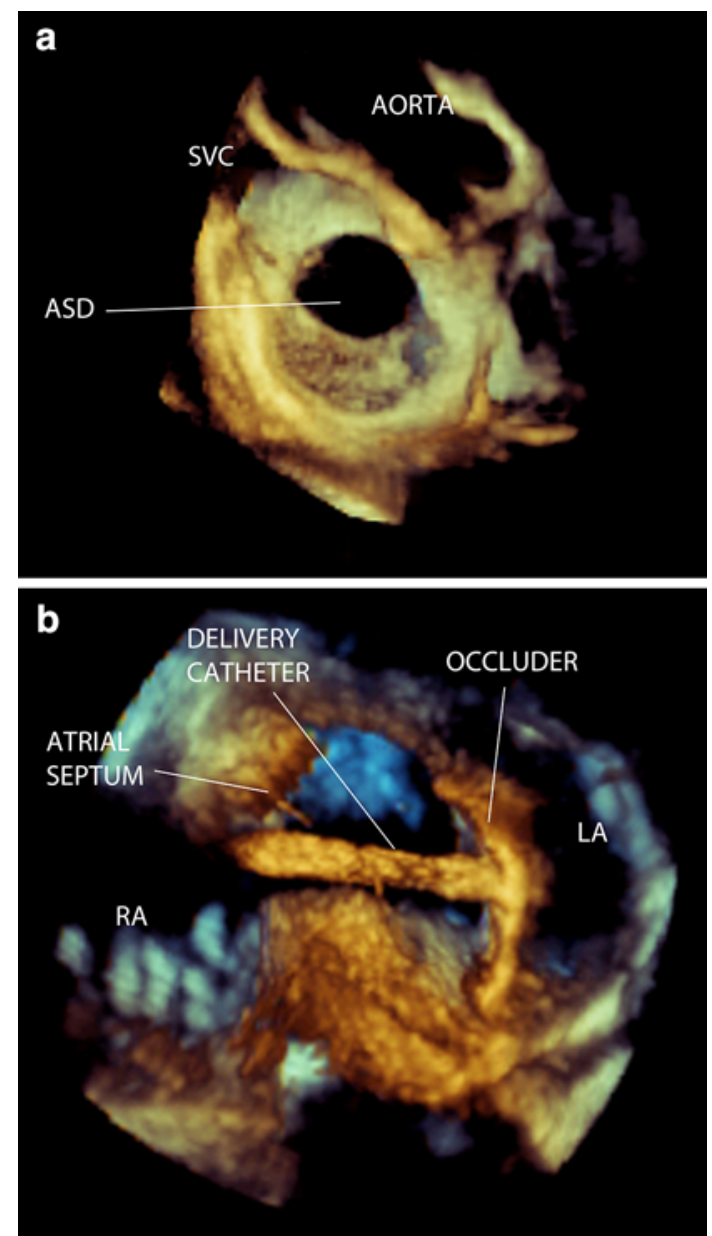

Fig. 1 3D TEE imaging of an atrial septal defected projected from the right atrial aspect (a). Live 3D echocardiography can also be employed to visualize the atrial septum, septal occlude and delivery catheter in a single projection (b)

congenital perimembranous VSD's and post infarct VSD's [26].

Imaging during catheter interventions has become a major role of real-time 3DE, not only in congenital heart disease practice but also in the guidance of multiple other procedures including trans-septal puncture, occlusion of paravalvular leaks, atrial appendage occlusion, mitral valve clipping, and transcatheter implantation of aortic valves. The ability to view catheter position, devices, and complex spatial orientation from a single live projection, typically viewed from a left atrium, has meant that $3 \mathrm{DE}$ is now firmly established in clinical practice. There are some limiting factors, which include the current size of 3D TEE probes that are unsuitable for use in children weighing less than $25-30 \mathrm{~kg}$ and temporal resolution that can become a significant factor where a large field of view is selected. Previous limitations such as lack of color flow Doppler for live $3 \mathrm{D}$ modalities and the inability to make distance or 
area measurements on rendered 3D images have been addressed in more recent software releases.

\section{Fetal Cardiology}

The use of 3DE during fetal life poses a number of technical challenges related to the small size of the fetal heart, fast heart rates, unpredictable acoustic window, and absence of an electrocardiographic signal to gate the echocardiographic images. In current practice, the most common type of $3 \mathrm{DE}$ performed during fetal life is achieved using spatio-temporal image correlation (STIC). This involves a slow sweep of the area of interest with multiple cross-sectional slices that are then reconstructed into a 3D image using algorithms which recognize the rhythmicity of the heart. The STIC technique and analysis software make a few assumptions with respect to ventricular shape; however, the major hindrance is the time taken to acquire such images (typically 7-15 s) with the potential for motion artifact. Recent studies have compared STIC images to those obtained using 3D matrix probes [27], with similar image quality but the avoidance of artefacts using the real-time 3DE approach. Within the past 1-2 years several groups have reported on the use of the STIC technique to produce reference ranges for cardiac structures [28, 29], ventricular volumes, ejection fraction (EF), and cardiac output [30, 31]. Recent work has made ingenious use of the "cross plane" imaging modality afforded by matrix transducers to enhance imaging of the fetal outflow tracts [32, 33]. Although not truly 3D, the technique has the advantage of simplicity and lack of artifact. With respect to prenatal diagnosis of congenital heart defects, the addition of $3 \mathrm{D}$ information provided additional information in around $6 \%$ of cases overall [34]. This relatively cautious conclusion is important as it emphasises that, currently, core fetal echocardiographic findings continue to be made by cross-sectional imaging, supplemented by color flow and other Doppler modalities. A recent multicenter study posed a different question concerning whether interrogation of a $3 \mathrm{D}$ volume at a remote center would provide sufficient information to make a full diagnosis of the congenital heart lesions [35]. This study confirmed that reinterrogation remains operator dependent and does not always provide full information. Operator confidence in their diagnosis did not correlate with the accuracy based on full pathological or postnatal examination.

\section{Volumetrics and Function}

The CMRI has been regarded as the "gold standard" for assessment of ventricular volumes and EF for some time.
However, CMRI remains expensive, has a requirement for sedation or anaesthesia in younger patients, and post processing of ventricular volumes remains time consuming. Thus, 3DE has been evaluated as an alternative for the assessment of the RV and LV in congenital heart disease. There has been good correlation in a direct comparison between 3DE and CMRI to assess RV volumes and EF in patients with congenital heart disease [36]. Importantly, 3DE has been further demonstrated to have good test-retest variability when applied to healthy subjects and patients with congenital heart disease [37]. The 3DE assessment of the RV has largely been focused on transthoracic echocardiography (TTE) image datasets. This has recently been extended to TEE imaging in the perioperative environment showing good agreement between TTE and TEE [38], offering the potential to improve quantitative evaluation of RV function during cardiac surgery.

However, 3DE derived RV volumes and EF continue to underestimate true values compared to CMRI [36, 39, 40, $41 \bullet, 42]$. Assessments of volumes in the RV remain difficult due to the constraints of altered ventricular geometry in these cases. This is compounded by the relatively poor spatial resolution of 3DE compared to CMRI, the anterior position of the RV resulting in incomplete coverage of the $\mathrm{RV}$ and poor endocardial border definition of the heavily trabeculated RV. Newer approaches are being tried to mitigate these challenges.

Knowledge based 3D reconstruction (3DR) is a novel technique that has been shown to produce accurate assessment of RV volumes and EF compared to CMR in patients with tetralogy of Fallot [43]. This approach uses a 2DE-based reconstruction method for quantifying volumes, which is achieved by acquisition of multiple standard cross-sectional views using a magnetic tracker system. The $\mathrm{RV}$ volumes are reconstructed using a database of $\mathrm{RV}$ shapes and sizes compiled from patients after tetralogy of Fallot repair [41•, 43]. Although currently validated for tetralogy of Fallot, this method has the potential for application in other forms of congenital heart disease and appears to agree more closely with MRI volumes than realtime 3DE.

The LV is less constrained by complexities of geometry. Assessment of LV volume, mass, and function has been found to correlate well to CMRI measurements when applied to young children under 4 years of age [44]. There was good comparable data even in small volume ventricles, although this study was performed under general anaesthesia with regulation of breathing. The authors employed a manual summation of discs methodology using 3D software and 4-beat acquisitions. The availability of newer software with single-beat acquisitions has the potential to capture good datasets for volumetric assessment, but this has yet to be tested. 


\section{Remodelling}

Despite some of the limitations of 3DE described above, 3D volume assessments have been applied to increase our understanding of ventricular remodelling in congenital heart disease. Van der Hulst et al. [45•] demonstrated changes in right ventricular remodelling post tetralogy of Fallot repair compared to controls, with the biggest change in ventricular volume seen in the apical trabecular region. The EF was preserved in this region, but significantly impaired in the inlet and outlet components. The reduced function in the RV outlet affected by patch placement and surgical scarring has been previously shown to relate to outcome. These data provide useful insight into the characteristic remodelling seen in post surgical repair in tetralogy of Fallot and allow for segmental analysis in the longitudinal assessment of these patients.

Remodelling in hypoplastic left heart syndrome (HLHS) has also been described using serial changes in right ventricular volume and function assessment prior to Stage 1 Norwood surgery through to post Norwood Stage 2 surgery [46]. Commercially available 3D RV function software was used to post process 3D datasets. Despite the geometrical variation of the systemic RV in HLHS, that study reported an increase in RV end diastolic volume indexed to body surface area (iEDV) and reduction in RV EF through the stages of HLHS surgical palliation. These findings are at odds with CMRI data from our unit, which show a reduction in RV iEDV post stage 2 [47]. This might be explained by the limitation of 3DE volumetric assessment and segmentation of univentricular hearts with a systemic RV using currently available commercial software.

\section{Flow}

Assessment of flow using 2DE is accepted to be prone to error [48]. Pulsed wave Doppler derived flow quantification relies on the velocity profiles across the valve being flat and uniform and a constant geometry and area across the valve during the cardiac cycle. Echocardiographic assessment of flow has the potential to quantify cardiac output, shunts, and regurgitant fractions. The 3DE applications to quantify these variables in clinical practice have, to date, been quite limited.

Mitral valve regurgitant fractions have recently been estimated using single-beat real time 3D color Doppler in patients with degenerative mitral valve disease [49]. Application of 3DE permitted direct measurement of the proximal isovelocity surface area (PISA) area without the need for geometric assumptions in contrast to 2DE. The 3DE of multiple vena contracta areas in assessment of mitral regurgitation has also been applied in 3D TEE with good agreement with thermodilution assessments [50]. These methods have the potential for application in congenital lesions where residual atrioventricular valve regurgitation is clinically important.

Automated 3DE quantification of flow is now possible with a recent publication demonstrating feasibility and accuracy of aortic stroke volume and mitral inflow volume measurement using a single-beat TTE acquisition [51•]. The field of view was sufficient to encompass both regions within a single dataset at a temporal resolution of 33 frames/s. The algorithm included automated detection of the LV endocardial border, mitral annulus, and left ventricular outflow tract. Mitral inflow and aortic stroke volumes were not statistically different from CMRI derived values. This application has the potential for important flow quantification in the assessment of congenital heart disease. However, it should be emphasized that the data reported recently related to adult patients without structural abnormality. The place of this technique to measure ventricular inflow and output in patients with congenital heart disease remains to be established.

\section{Deformation Imaging}

Myocardial strain and strain rate allow for a quantifiable, objective measure of global or regional myocardial deformation. The 3DE myocardial strain has major attractions including angle independence and reduction of strain estimation errors related to out of plane myocardial motion [52॰]. Furthermore, 3DE techniques can incorporate the ventricular length to facilitate measurement of the left ventricular torsion. However, 3DE deformation imaging still suffers from variability in algorithms and deformation parameters between vendors $[52 \bullet, 53]$. This area was the subject of an excellent recent review [52॰]. Therefore, validity of each algorithm needs to be assessed before these are applied in clinical practice. There is now a joint working group of the American Society of Echocardiography and European Association of Cardiovascular Imaging to address the issue of standardization in 2DE strain imaging as an initial measure.

\section{Technical Advances}

Despite improvements in image quality with higher frequency matrix array transducers and quicker processing ability, significant limitations of 3DE in congenital heart disease relate to having an adequate field of view, high temporal resolution, and good endocardial border definition. New methods of image registration and fusion of multiple 3D images using feature consistency in our own 
research facility has been shown to improve the field of view, image quality, and endocardial border definition, with lower noise from the ventricular cavity [54]. This work has been extended to assess flows using multiple registered echocardiographic Doppler views [55•]. In contrast to previous echocardiographic multi-view approaches, we were able to reconstruct a full 3D flow field with good accuracy across the aortic and mitral valves. This has the potential for quantification and demonstration of flow mechanics in chambers of non-uniform geometry, which is pertinent to patients with congenital cardiac malformations. Image registration methods which are currently limited by the need for offline post processing are being modified to offer real time registration [56]. However, this is still in an early pre-clinical setting.

There have also been developments in the application of $3 \mathrm{DE}$ to guide catheter interventions where fluoroscopic information is also required. Registration of $3 \mathrm{DE}$ to X-ray using image based electromagnetic probe tracking allows simultaneous real time visualization of cardiac anatomy and interventional devices. The registration error between the two modalities is low [57]. This technique is now being evaluated in clinical practice, guiding catheter positioning within a 3DE volume [58] during cardiac catheterization. This has the potential application to assist during complex interventions, including ablation therapy, in congenital heart disease.

Advances in procedural 3DE imaging have not been restricted to the catheterization laboratory. One of the issues affecting 3DE in the operating room has been the relatively low frame rates of real-time 3DE particularly for fast-moving valvar structures. A novel technique for enhancing 3D frame rates has recently been described, albeit with a longer acquisition time than current techniques [59]. Furthermore, with the development of robotic surgery, $3 \mathrm{DE}$ is finding a central role in imaging of such procedures [60•].

Automation methods for post processing [61] remain crucial to bringing $3 \mathrm{DE}$ techniques into clinical practice. One of the improvements suggested is applying a population-based method for quantification using actual patient measurements to build a disease specific database. This is particularly relevant in congenital heart disease with altered geometries. This approach has been used in knowledge based reconstruction methods using 2DE [43] but can be applied to 3DE to allow for smoother segmentation and volumetric assessments.

\section{Conclusion}

The quality of 3DE and scope of application of this imaging modality is increasing rapidly. Key areas remain standardization, validation, and reproducibility of the technique. Critical evaluation of the added value of $3 \mathrm{DE}$ and impact on patient outcome needs to continue to parallel technical advances.

Disclosure John M. Simpson has received travel/accommodations expenses covered or reimbursed from Philips Medical Systems. Kuberan Pushparajah declares no conflicts of interest.

\section{References}

Papers of particular interest, published recently, have been highlighted as:

- Of importance

1. Zhang L, Xie M, Balluz R, Ge S. Real time three-dimensional echocardiography for evaluation of congenital heart defects: state of the art. Echocardiography. 2012;29:232-41.

2. Shirali GS. Three-dimensional echocardiography in congenital heart disease. Echocardiography. 2012;29:242-8.

3. Simpson JM, Miller O. Three-dimensional echocardiography in congenital heart disease. Arch Cardiovasc Dis. 2011;104:45-56.

4. - Simpson J, Miller O, Bell A, Bellsham-Revell H, McGhie J, Meijboom F. Image orientation for three-dimensional echocardiography of congenital heart disease. Int J Cardiovasc Imaging. 2012;28:743-53. This paper presents guidance for standardization of image orientation and presentation in $3 D E$ as is practised at these authors' institution.

5. - Lang RM, Badano LP, Tsang W, et al. EAE/ASE recommendations for image acquisition and display using three-dimensional echocardiography. J Am Soc Echocardiogr. 2012;25:3-46. This paper presents the joint recommendations of the European Association of Echocardiography and the American Society of Echocardiography for standardization of image orientation and presentation in $3 D E$.

6. Anderson RH, Razavi R, Taylor AM. Cardiac anatomy revisited. J Anat. 2004;205(3):159-77.

7. Faletra FF, Nucifora G, Ho SY. Real-time 3-dimensional transesophageal echocardiography of the atrioventricular septal defect. Circ Cardiovasc Imaging. 2011;4:e7-9.

8. Kutty S, Smallhorn JF. Evaluation of atrioventricular septal defects by three-dimensional echocardiography: benefits of navigating the third dimension. J Am Soc Echocardiogr. 2012;25:932-44.

9. Takahashi K, Mackie AS, Thompson R, et al. Quantitative realtime three-dimensional echocardiography provides new insight into the mechanisms of mitral valve regurgitation post-repair of atrioventricular septal defect. J Am Soc Echocardiogr. 2012;25: 1231-44.

10. Valverde I, Rawlins D, Austin C, Simpson JM. Three-dimensional echocardiography in the management of parachute mitral valve. Eur Heart J Cardiovasc Imaging. 2012;13:446.

11. Anwar AM, Nosir YF, Galal AN, Al-Barakati M, Chamsi-Pasha $\mathrm{H}$. Incremental utility of real time three-dimensional tranthoracic echocardiography in the assessment of congenitally malformed aortic valve. Echocardiography. 2012;29:978-83.

12. Sadron Blaye-Felice MA, Seguela PE, Arnaudis B, Dulac Y, Lepage B, Acar P. Usefulness of three-dimensional transthoracic echocardiography for the classification of congenital bicuspid aortic valve in children. Eur Heart J Cardiovasc Imaging. 2012; 13:1047-52. 
13. Noel CV, Choy RM, Lester JR, Soriano BD. Accuracy of matrixarray three-dimensional echocardiographic measurements of aortic root dilation and comparison with two-dimensional echocardiography in pediatric patients. J Am Soc Echocardiogr. 2012;25:287-93.

14. Black D, Ahmad Z, Lim Z, Salmon A, Veltdman G, Vettukattil J. The accuracy of three-dimensional echocardiography with multiplanar reformatting in the assessment of the aortic valve annulus prior to percutaneous balloon aortic valvuloplasty in congenital heart disease. J Invasive Cardiol. 2012;24:594-8.

15. Bharucha T, Fernandes F, Slorach C, Mertens L, Friedberg MK. Measurement of effective aortic valve area using three-dimensional echocardiography in children undergoing aortic balloon valvuloplasty for aortic stenosis. Echocardiography. 2012;29: 484-91.

16. Pushparajah K, Barlow A, Tran VH, et al. A systematic threedimensional echocardiographic approach to assist surgical planning in double outlet right ventricle. Echocardiography. 2012.

17. Bilska KM, Kehrens CM, Riley G, Anderson RH, Marek J. Subcostal real-time three-dimensional echocardiography of interatrial communications: reconstruction of an oval fossa defect, a superior sinus venosus defect with partially anomalous pulmonary venous drainage, an infero-posterior oval fossa defect, and a coronary sinus defect. Cardiol Young. 2012;22:145-51.

18. Vaidyanathan B, Simpson JM, Kumar RK. Transesophageal echocardiography for device closure of atrial septal defects: case selection, planning, and procedural guidance. JACC Cardiovasc Imaging. 2009;2:1238-42.

19. Pushparajah K, Miller OI, Simpson JM. 3D echocardiography of the atrial septum: anatomical features and landmarks for the echocardiographer. JACC Cardiovasc Imaging. 2010;3:981-4.

20. Seo JS, Song JM, Kim YH, et al. Effect of atrial septal defect shape evaluated using three-dimensional transesophageal echocardiography on size measurements for percutaneous closure. J Am Soc Echocardiogr. 2012;25:1031-40.

21. Garcia-Fuertes D, Mesa-Rubio D, Ruiz-Ortiz M, et al. Monitoring complex secundum atrial septal defects percutaneous closure with real time three-dimensional echocardiography. Echocardiography. 2012;29:729-34.

22. Roberson DA, Cui W, Patel D, et al. Three-dimensional transesophageal echocardiography of atrial septal defect: a qualitative and quantitative anatomic study. J Am Soc Echocardiogr. 2011;24:600-10.

23. Wang L, Cao S, Li J, et al. Transcatheter closure of congenital perimembranous ventricular septal defect in children using symmetric occluders: an 8-year multiinstitutional experience. Ann Thorac Surg. 2012;94:592-8.

24. Bass JL, Gruenstein D. Transcatheter closure of the perimembranous ventricular septal defect-preclinical trial of a new Amplatzer device. Catheter Cardiovasc Interv. 2012;79:1153-60.

25. Sivakumar K, Singhi A, Pavithran S. Enface Reconstruction of VSD on RV Septal Surface Using Real-Time 3D Echocardiography. JACC Cardiovasc Imaging. 2012;5:1176-80.

26. Charakida M, Qureshi S, Simpson J. 3D Echocardiography for Planning and Guidance of Interventional Closure of Ventricular Septal Defects. JACC Cardiovasc Imaging. 2013;6(1):120-3.

27. Xiong Y, Liu T, Wu Y, et al. Comparison of real-time threedimensional echocardiography and spatiotemporal image correlation in assessment of fetal interventricular septum. J Matern Fetal Neonatal Med. 2012;25:2333-8.

28. Luewan S, Yanase Y, Tongprasert F, Srisupundit K, Tongsong T. Fetal cardiac dimensions at 14-40 weeks' gestation obtained using cardio-STIC-M. Ultrasound Obstet Gynecol. 2011;37:416-22.

29. Tongprasert F, Srisupundit K, Luewan S, et al. Reference ranges of fetal aortic and pulmonary valve diameter derived by STIC from 14 to 40 weeks of gestation. Prenat Diagn. 2011;31:439-45.
30. Simioni C, Nardozza LM, Araujo Junior E, et al. Heart stroke volume, cardiac output, and ejection fraction in 265 normal fetus in the second half of gestation assessed by $4 \mathrm{D}$ ultrasound using spatio-temporal image correlation. J Matern Fetal Neonatal Med. 2011;24:1159-67.

31. Schoonderwaldt EM, Groenenberg IA, Hop WC, Wladimiroff JW, Steegers EA. Reproducibility of echocardiographic measurements of human fetal left ventricular volumes and ejection fractions using four-dimensional ultrasound with the spatiotemporal image correlation modality. Eur J Obstet Gynecol Reprod Biol. 2012;160:22-9.

32. Xiong Y, Chen M, Chan LW, et al. Scan the fetal heart by realtime three-dimensional echocardiography with live xPlane imaging. J Matern Fetal Neonatal Med. 2012;25:324-8.

33. Xiong Y, Chen M, Chan LW, et al. A novel way of visualizing the ductal and aortic arches by real-time three-dimensional ultrasound with live xPlane imaging. Ultrasound Obstet Gynecol. 2012;39:316-21.

34. Yagel S, Cohen SM, Rosenak D, et al. Added value of three-/ four-dimensional ultrasound in offline analysis and diagnosis of congenital heart disease. Ultrasound Obstet Gynecol. 2011;37: $432-7$.

35. Adriaanse BM, Tromp CH, Simpson JM, et al. Interobserver agreement in detailed prenatal diagnosis of congenital heart disease by telemedicine using four-dimensional ultrasound with spatiotemporal image correlation. Ultrasound Obstet Gynecol. 2012;39:203-9.

36. van der Zwaan HB, Helbing WA, McGhie JS, et al. Clinical value of real-time three-dimensional echocardiography for right ventricular quantification in congenital heart disease: validation with cardiac magnetic resonance imaging. J Am Soc Echocardiogr. 2010;23:134-40.

37. van der Zwaan HB, Geleijnse ML, McGhie JS, et al. Right ventricular quantification in clinical practice: two-dimensional vs. threedimensional echocardiography compared with cardiac magnetic resonance imaging. Eur J Echocardiogr. 2011;12:656-64.

38. Fusini L, Tamborini G, Gripari P, et al. Feasibility of intraoperative three-dimensional transesophageal echocardiography in the evaluation of right ventricular volumes and function in patients undergoing cardiac surgery. J Am Soc Echocardiogr. 2011;24: 868-77.

39. Crean AM, Maredia N, Ballard G, et al. 3D Echo systematically underestimates right ventricular volumes compared to cardiovascular magnetic resonance in adult congenital heart disease patients with moderate or severe RV dilatation. J Cardiovasc Magn Reson. 2011;13:78.

40. Grewal J, Majdalany D, Syed I, Pellikka P, Warnes CA. Threedimensional echocardiographic assessment of right ventricular volume and function in adult patients with congenital heart disease: comparison with magnetic resonance imaging. J Am Soc Echocardiogr. 2010;23:127-33.

41. - Dragulescu A, Grosse-Wortmann L, Fackoury C, Mertens L. Echocardiographic assessment of right ventricular volumes: a comparison of different techniques in children after surgical repair of tetralogy of Fallot. Eur Heart J Cardiovasc Imaging. 2012;13:596-604. This is a comparison of $R V$ volumeteric assesments using a novel 2DE technique with knowledge based $3 D$ reconstruction versus $3 D E$ volume acquisition analysis and CMRI.

42. Khoo NS, Young A, Occleshaw C, Cowan B, Zeng IS, Gentles TL. Assessments of right ventricular volume and function using three-dimensional echocardiography in older children and adults with congenital heart disease: comparison with cardiac magnetic resonance imaging. J Am Soc Echocardiogr. 2009;22:1279-88.

43. Dragulescu A, Grosse-Wortmann L, Fackoury C, et al. Echocardiographic assessment of right ventricular volumes after 
surgical repair of tetralogy of fallot: clinical validation of a new echocardiographic method. J Am Soc Echocardiogr. 2011;24: 1191-8.

44. Friedberg MK, Su X, Tworetzky W, Soriano BD, Powell AJ, Marx GR. Validation of 3D echocardiographic assessment of left ventricular volumes, mass, and ejection fraction in neonates and infants with congenital heart disease: a comparison study with cardiac MRI. Circ Cardiovasc Imaging. 2010;3:735-42.

45. - van der Hulst AE, Roest AA, Holman ER, et al. Real-time three-dimensional echocardiography: segmental analysis of the right ventricle in patients with repaired tetralogy of fallot. J Am Soc Echocardiogr. 2011;24:1183-90. This paper presents a method and results of segmentation of the right ventricle to permit separate evaluation of the inlet, apical trabecular and outflow segments of the right ventricle.

46. Kutty S, Graney BA, Khoo NS, et al. Serial assessment of right ventricular volume and function in surgically palliated hypoplastic left heart syndrome using real-time transthoracic threedimensional echocardiography. J Am Soc Echocardiogr. 2012; 25:682-9.

47. Bellsham-Revell HR, Tibby SM, Bell AJ et al. Serial magnetic resonance imaging in hypoplastic left heart syndrome gives valuable insight into ventricular and vascular adaptation. J Am Coll Cardiol. 2013;61(5):561-70.

48. Ge S. Automated Measurement of Stroke Volumes by Real-Time Three-Dimensional Doppler Echocardiography: coming of Age? J Am Soc Echocardiogr. 2012;25:66-7.

49. de Agustín JA, Marcos-Alberca P, Fernandez-Golfin C, et al. Direct Measurement of Proximal Isovelocity Surface Area by Single-Beat Three-Dimensional Color Doppler Echocardiography in Mitral Regurgitation: a Validation Study. J Am Soc Echocardiogr. 2012;25:815-23.

50. Hyodo E, Iwata S, Tugcu A, et al. Direct Measurement of Multiple Vena Contracta Areas for Assessing the Severity of Mitral Regurgitation Using 3D TEE. JACC Cardiovasc Imaging. 2012;5:669-76.

51. - Thavendiranathan P, Liu S, Datta S, et al. Automated Quantification of Mitral Inflow and Aortic Outflow Stroke Volumes by Three-Dimensional Real-Time Volume Color-Flow Doppler Transthoracic Echocardiography: comparison with Pulsed-Wave Doppler and Cardiac Magnetic Resonance Imaging. J Am Soc Echocardiogr. 2012;25:56-65. Feasibility and accuracy data of a novel automated 3DE quantification of aortic stroke volume and mitral inflow volume measurement using a single beat TTE acquisition.

52. - Jasaityte R, Heyde B, D'hooge J. Current State of ThreeDimensional Myocardial Strain Estimation Using Echocardiography. J Am Soc Echocardiogr. 2012. An updated comprehensive review of $3 D E$ myocardial strain estimation.

53. Gayat E, Ahmad H, Weinert L, Lang RM, Mor-Avi V. Reproducibility and Inter-Vendor Variability of Left Ventricular Deformation Measurements by Three-Dimensional SpeckleTracking Echocardiography. J Am Soc Echocardiogr. 2011;24: 878-85.

54. Yao C, Simpson JM, Schaeffter T, Penney GP. Multi-view 3D echocardiography compounding based on feature consistency. Phys Med Biol. 2011;56:6109-28.

55. • Gomez A, Simpson J, Cheng Y, Schaeffter T, Penney G. 3D flow reconstruction from multiple registered echo Doppler views. In: 2011 IEEE international symposium on biomedical imaging: from nano to macro; 2011.p. 879-82. This is a pre-clinical technical paper demonstrating the feasibility of angle-independent $3 D E$ derived flow reconstruction from multiple fused $3 D E$ colour Doppler views.

56. Schneider RJ, Perrin DP, Vasilyev NV, Marx GR, Del Nido PJ, Howe RD. Real-time image-based rigid registration of threedimensional ultrasound. Med Image Anal. 2012;16:402-14.

57. Gao G, Penney G, Ma Y, et al. Registration of 3D trans-esophageal echocardiography to X-ray fluoroscopy using image-based probe tracking. Med Image Anal. 2012;16:38-49.

58. Housden RJ, Arujuna A, Ma Y et al. Evaluation of a real-time hybrid three-dimensional echo and x-ray imaging system for guidance of cardiac catheterisation procedures. Proceedings of the 15th international conference on Medical Image Computing and Computer-Assisted Intervention-Volume Part II. Nice. France: Springer; 2012. p. 25-32.

59. Vasilyev NV, Dupont PE, del Nido PJ. Robotics and imaging in congenital heart surgery. Future Cardiol. 2012;8:285-96.

60. • Perrin DP, Vasilyev NV, Marx GR, del Nido PJ. Temporal enhancement of 3D echocardiography by frame reordering. JACC Cardiovasc Imaging. 2012;5:300-4. This paper presents a method for vastly increasing the temporal resolution of $3 D E$ to permit optimal visualization of fast moving structures such as atrioventricular valves.

61. Leung KY, Bosch JG. Automated border detection in threedimensional echocardiography: principles and promises. Eur J Echocardiogr. 2010;11:97-108 